\title{
Physiological and Cultural Properties of the Luminous Fungus Omphalotus af. Illudent
}

\author{
Dao Thi Van* \\ BIO-LUMI Company Ltd., 2/6 Do Nang Te, \\ Binh Tri Dong ward Binh Tan dist, Ho Chi Minh, Vietnam ${ }^{1}$
}

Received 1.06.2009, received in revised form 8.06.2009, accepted 15.06.2009

\begin{abstract}
The luminous fungus that we have described as a presumably new species, Omphalotus af. illudent, grows in rain forests in the south of Vietnam on the dead tree wood, mostly on the rubber tree (Hevea). It emits exceptionally bright light in all phases of its life cycle, from germinate spores and mycelium to fruiting bodies. This study describes the life cycle of Omphalotus af. illudent (Neonatapanus) and determination of the optimal parameters of the temperature, humidity, $\mathrm{pH}$, concentration of mineral elements, and composition of the nutrient media. Measurements have been performed to determine the destructive enzymatic activity of the mycelium towards some of the wood components. Optimal mycelial growth and luminescence have been found to occur under different conditions. The study of the parameters of Omphalotus af.illudent provided a basis for successful large-scale cultivation of this fungus and, hence, preparation of sufficient quantities of material for studies of the biochemical and genetic mechanisms underlying fungal bioluminescence and examination of the biochemical components of the fungus that are potentially valuable for pharmacology (illudin etc.). The brightness and steadiness of Omphalotus af. illudent luminescence make this fungus a good candidate for continuous bioluminescent analytical systems.
\end{abstract}

Keywords: fungi bioluminescence, Omphalotus af. illudent, fungi cultivation, bioluminescence

\section{Introduction}

Of the three kingdoms of living nature, fungi are the least understood of all. They wonderfully combine properties of animals and plants, being, at the same time, equidistant from them. This makes the study of fungi an exceptionally fascinating scientific challenge. The power and sophistication of the contemporary analytical methods have to bring success to researchers in this little-studied field. Moreover, the practical potential of fungi is enormous: suffice it to say that fungi, although lower ones, gave rise to the era of antibiotics. The potentials of the higher fungi in this and in many other respects, except maybe gastronomic and toxicological ones, have not been studied by methods of molecular biology, genetics, and biophysics.

Among the obstacles are difficulties entailed in cultivating various species of higher fungi. Large-scale cultivation has been developed for just a few dozen edible or poisonous species of the whole spectrum of basidiomycetes. It is wellknown that mushroom hunting in the wild is an unreliable procedure. A profound study, however, requires fungal biomass that could be consistently produced and reproduced.

* Corresponding author E-mail address: van79bioluminescent@yahoo.com

(C) Siberian Federal University. All rights reserved 
Cultivation of fungi that form obligate mycorhizal associations with plants is obviously very difficult and yields poorly reproducible results. The formulations of the culture media for higher fungi are usually very complex and include, in addition to conventional energy sources and plastic materials, a number of specific substances necessary for the growth and morphogenesis of fungi: complex mixtures of organic substrates such as wood. This is suggestive of the high auxotrophy and narrow species specificity of their metabolism, which make their cultivation so difficult. On the other hand, their specific metabolism makes the discovery of new, potentially useful, metabolites a highly probable event.

An interesting natural phenomenon, which can also be used as an analytical tool, is the ability of just a few species of higher fungi to emit visible light. About 90 species of luminescent fungi have been found so far. They have been quite fully described in the recent review by Desjardin and his co-authors (2008). The genus Omphalotus (Omphalotaceae) has been rather reviewed by $\mathrm{M}$. Kirchmair et al. (2002), and at least 4 Omphalotus species were reported to be able to emit light. ${ }^{1}$ While the mechanism of bioluminescence of animals and bacteria has been understood quite well (Shimamura, 2006), the chemical basis of fungal luminescence remains understudied (Desjardin, 2008).

The source of luminescence in all light-emitting organisms, except fungi, is the classical reaction: oxidation of organic substrate by oxygen, necessarily involving the enzyme. The substrates have been named luciferins and the enzymes, correspondingly, luciferases. It is noteworthy that these are

Recently Omphalotus af. illudent was renamed as Neonatapanus nambi (Peterson, 1999) in agreement with genetic similarity (J. Bisset, 2009, personal communication) collective names, which denote the functions of their bearers in bioluminescent reaction rather than their chemical nature. Molecular basis of more than ten bioluminescent systems has been deciphered by now, showing that functions of luciferins and luciferases in them are performed by chemically different molecules (Shimomura, 2006). Bioluminescence is characterized by remarkably high quantum yields. In light-emitting insects, e.g., they are higher than $80 \%$ (Bitler, Mac Elroy, 1957). The high light efficiency of the reaction must be mainly determined by the properties of the enzyme - luciferase.

A special class is represented by the socalled precharged systems of coelenterates, which do not need oxygen during light emission. Yet, oxygen joins the luminescent system of coelenterates prior to luminescence and forms stable complexes with it.

Thus, oxygen must necessarily be part of all bioluminescence systems. They are all consistent with the classic experiment performed by R. Dubois (1885), who showed the necessity of the air oxygen and identified the two principal components of the reaction a thermo-stable component and a thermo-labile one. He named the former luciferin and the latter luciferase. These are the most general properties of bioluminescence reactions of animals and bacteria.

The bioluminescence system of fungi has one fundamental property in common with them - it is oxygen-dependent. The other fundamental property of all known bioluminescence systems the enzymatic mechanism - has not been proven for fungi.

O. Shimomura, who studied the bioluminescence system of the fungus Panellus stipticus, suggested that its luminescence does not involve any specific enzyme but is spontaneous aerobic chemiluminescence occurring due to 

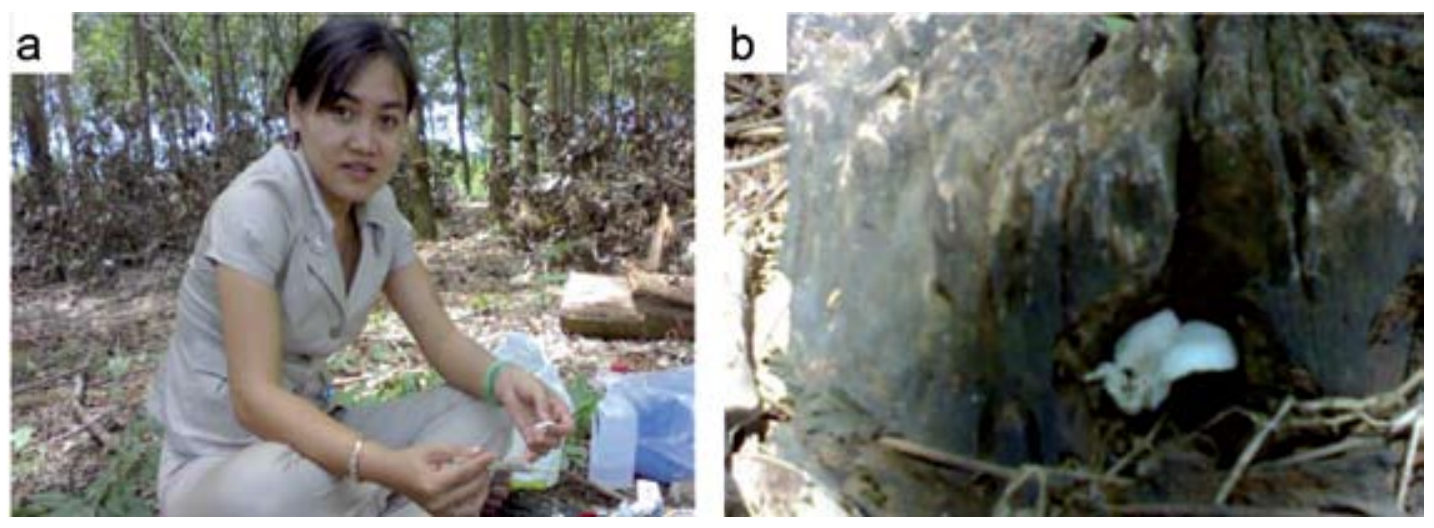

Fig. 1. a) Collection of the starting material for fungal culture in forest. b). Omphalotus af. illudent fruiting body on the rubber tree - its typical habitat

oxidation of the substrate, which he named panal (Shimomura, 1992), under the action of superoxide dismutase.

If this is true, luminosity in fungi is fundamentally different from the common mechanism of bioluminescence. However, contrary to Shimomura's conclusion, a team of researchers in Brazil (Desjardin et al., 2008) reported the discovery of the two-component luminescence system in the fungus Mycena $s p$. - the thermo-stable fraction (luciferin) and the thermo-labile one (luciferase) (Desjardin et al., 2005; 2007; Desjardin and Braga-Neto 2007).

Thus, until now fungal luminescence remains a natural phenomenon whose mechanism is not even basically understood.

In order to study it using the tools of contemporary molecular biology, genetics, and biophysics, it is first of all necessary to have a sustainable, reproducible laboratory culture of luminescent fungi, which can sustain bright luminescence throughout their life cycle.

The purpose of our work was to develop biotechnology for sustainable cultivation of the luminescent fungus Omphalotus af. illudent, whose luminescence is exceptionally bright in all phases of its life cycle. This communication describes the obtained results.
In the moist forest in the south-east of Vietnam, during the monsoon season, our attention was attracted by the mushroom emitting bright light, whose fruiting bodies grew on trunks of living Hevea ${ }^{2}$ trees and on dead wood. We studied relationships of the growth rate, biomass increase, development of fruiting bodies, and spore formation to the chemical composition of the nutrient medium, humidity, temperature, and lighting conditions.

\section{Objects and methods}

\section{Natural habitat of the luminous fungus}

We collected luminous fruiting-bodies for cultivation and research from high-land tropical forest in Lam Dong province, Da Lat, in the

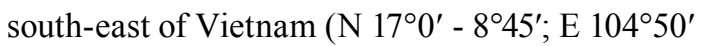
$-109^{\circ} 30^{\prime}$ ), annually from September to December (Fig. 1a). The site is a relatively cool high land with very many rubber trees (Hevea). Luminous fungi grow on rotten logs of dead rubber trunks and sometimes on dead cashew logs in the rain forest and under the shadow of other larger trees around (Fig. 1b).

Fig. 2 is presented photos of Omphalotus af. illudent fruiting bodies on the cross-section of the rubber tree trunk taken under ambient-

\footnotetext{
2 Hevea - the rubber tree (editor's note)
} 

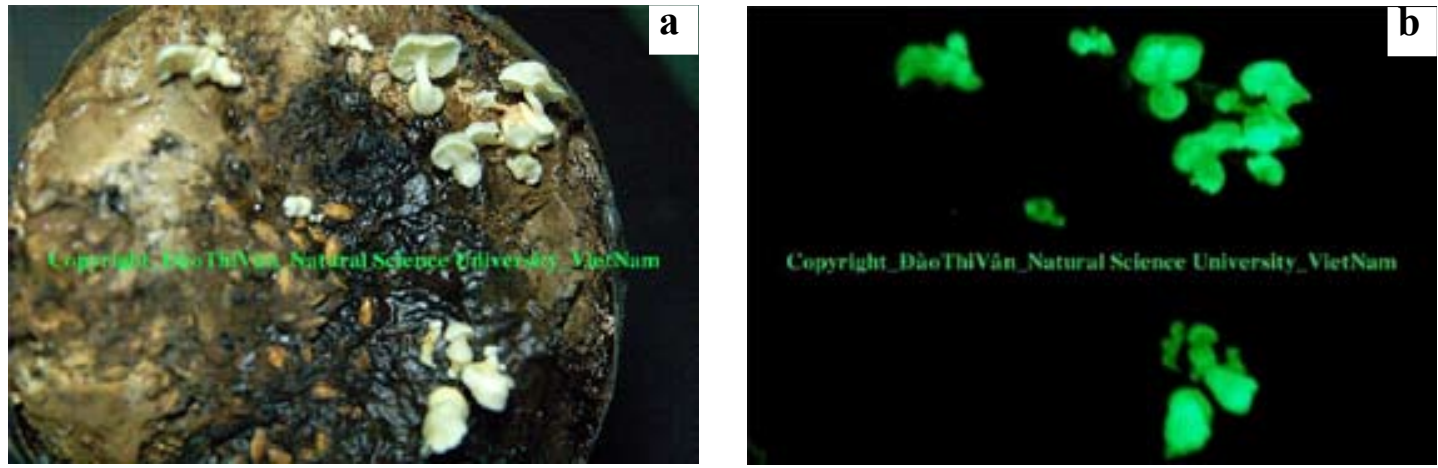

Fig. 2. Omphalotus af. illudent fruiting bodies on the cross-section of the rubber tree trunk: a) under ambient-light conditions, b) in the dark, in the light of the mushrooms' luminescence

light conditions and in the light of their own luminescence in the dark (the photos taken by the author).

\section{Cultivation methods}

The studied material was the culture of the fungus Omphalotus af. illudent that we grew from wild-collected Omphalotus af. illudent fruiting bodies.

In order to substantiate the biotechnology of cultivating Omphalotus af. Illudent, we studied various dependencies of growth, development, and luminescence of the fungus Omphalotus af. illudent in mycologically pure large-scale culture. We used the method of large-scale cultivation of edible mushrooms conventionally used in Vietnam, which we later modified taking into account specific properties of Omphalotus af. illudent and purposes of cultivation.

Cultivation was performed on benches, in closed, but not isolated from the environment, rooms with controlled humidity. Humidity was measured using a hygrometer («Hanna», Italy). The temperature was monitored. Light intensity was measured using a lux meter.

Luminescence of fungus samples was measured in relative units using a lux meter, manufactured in the workshop of the Ho Chi Minh City University.
We used follow nutrient media:

1) PG and PGA (Potato Glucose and Potato Glucose Agar): potato $200 \mathrm{~g}$; glucose $20 \mathrm{~g}$; agar $20 \mathrm{~g}$; water $1000 \mathrm{ml}$.

2) Hamada and Hamada agar: glucose $20 \mathrm{~g}$, yeast extract $5 \mathrm{~g}$; agar $15 \mathrm{~g}$; $1 \mathrm{~N} \mathrm{HCl} 1.6 \mathrm{ml}$; water $1000 \mathrm{ml}$.

3) Peptone and peptone agar : glucose 20 g; peptone $5 \mathrm{~g}$; yeast extract $2 \mathrm{~g}$; $\mathrm{KH}_{2} \mathrm{PO}_{4} 1 \mathrm{~g}$; $\mathrm{MgSO}_{4} 0.5 \mathrm{~g}$; agar $20 \mathrm{~g}$; water $1000 \mathrm{ml}$.

4) $Y M$ and YMA (Yeast Malt): glucose 10 $\mathrm{g}$; peptone $5 \mathrm{~g}$; yeast extract $3 \mathrm{~g}$; malt extract $3 \mathrm{~g}$; agar $20 \mathrm{~g}$, water $1000 \mathrm{ml}$.

5) $\mathrm{M}$ and MA (Malt): glucose $2 \mathrm{~g}$; malt extract $20 \mathrm{~g}$; peptone $1 \mathrm{~g}$; agar $20 \mathrm{~g}$; water 1000 $\mathrm{ml}$.

6) Wakman: glucose $20 \mathrm{~g}$; peptone $5 \mathrm{~g}$; $\mathrm{KH}_{2} \mathrm{PO}_{4} 1 \mathrm{~g} ; \mathrm{MgSO}_{4} 0.5 \mathrm{~g}$; agar $20 \mathrm{~g}$; water 1000 $\mathrm{ml}$.

7) Chang : glucose $20 \mathrm{~g}$; peptone $2 \mathrm{~g}$; $\mathrm{KH}_{2} \mathrm{PO}_{4} 0.46 \mathrm{~g} ; \mathrm{K}_{2} \mathrm{HPO}_{4} 1 \mathrm{~g} ; \mathrm{MgSO}_{4} 0.5 \mathrm{~g}$; agar $20 \mathrm{~g}$; thiamine $0.5 \mathrm{mg}$; water $1000 \mathrm{ml}$.

Biomass increase was measured in the liquid media PG, H, P, YM, M; the linear rate of mycelium growth was measured in the cultures on agar. The biomass of fruiting bodies was air dried in the thermostat at the temperature not higher than $35^{\circ} \mathrm{C}$.

In experiments with different culture parameters each variant was measured at least in 

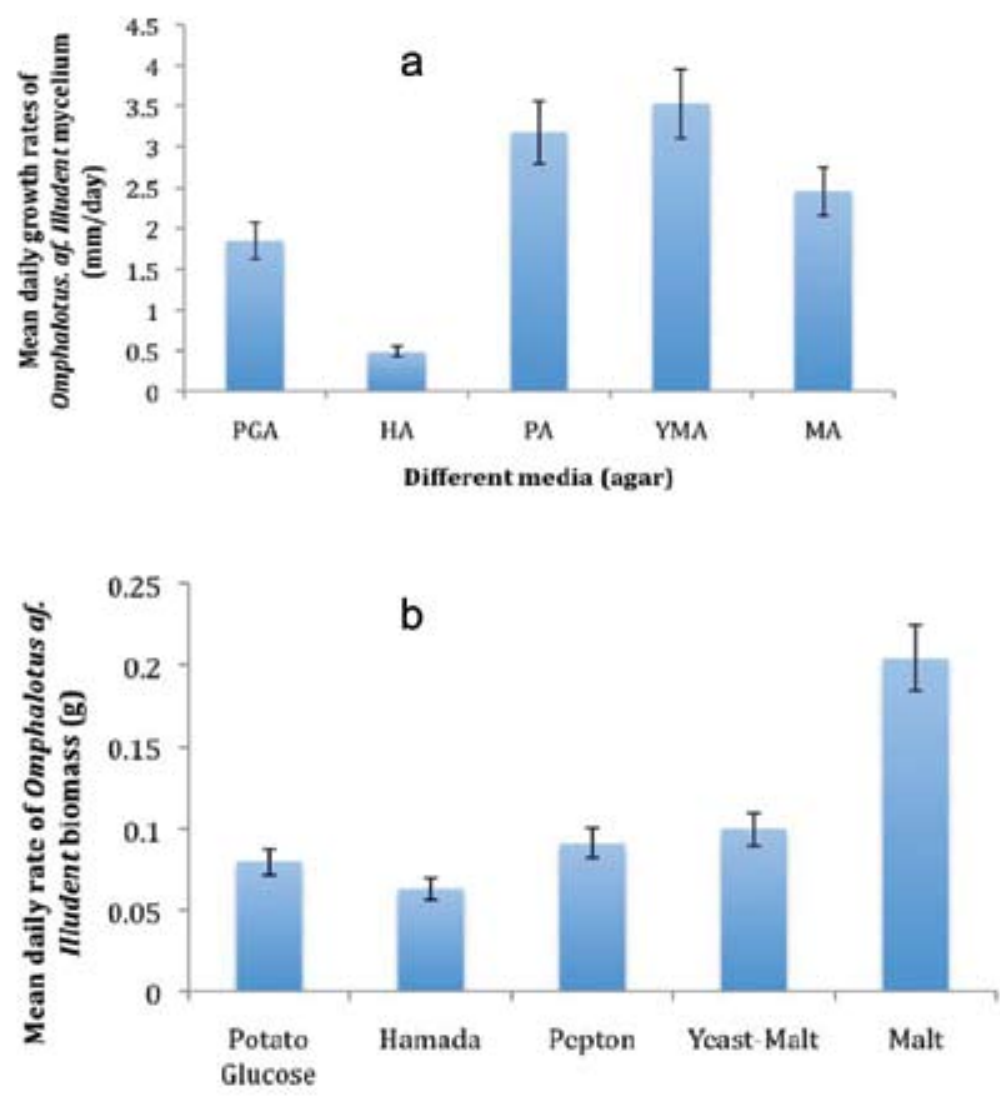

Different media (liquid)

Fig. 3. Mean daily growth rates of Omphalotus af. Illudent on different media: a) mycelium size, b) biomass

three replicates, with the statistical processing of the initial measurement results.

\section{Results and Discussion}

\section{Trial-and-error selection}

of the nutrient medium for Omphalotus af. illudent

Mycelium growth was measured based on two parameters - linear rate of hyphal growth over the surface of the solid nutrient medium, $\mathrm{mm}$ per day, and increase in the fresh biomass of growing fungus in the liquid culture, mg per day. The results of daily measurements averaged over three replicates. The growth rates on agar averaged over 8 days are presented in Fig. 3a, and diagrams of the mean daily rate of biomass increase in liquid culture are given in Fig.3b.
Interestingly, the sequences in the rows of diagrams of the linear growth rate and biomass increase are not quite similar.

The highest rate of linear growth was registered on YMA, while the fastest biomass increase - on $M$, which is different from $\mathrm{YM}$ in that it contains no yeast extract. The lowest growth and biomass increase rates of Omphalotus af. illudent were registered on Hamada medium.

Thus, the processes requiring the highest proliferation rate of Omphalotus af. illudent mycelium should be performed using YMA medium; however, fungus biomass increases fastest on the $M$ medium. Moreover, the difference is quite significant: the linear rate of mycelium growth on YMA is $20 \%$ higher than 


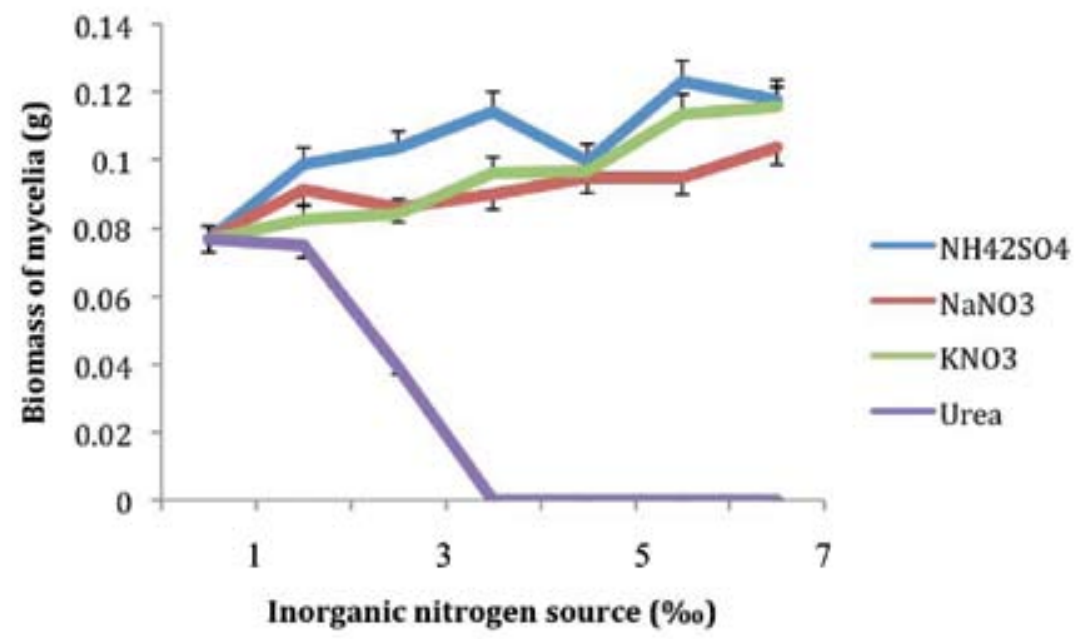

Fig. 4. Relationship of Omphalotus af. illudent biomass increase to the form of nitrogen nutrition

on MA. On the other hand, biomass increase rate on $\mathrm{M}$ is more than twice higher than on YM.

Apparently, the yeast extract contains a factor that slows down the buildup of Omphalotus af. illudent biomass, although it rather stimulates than hinders mycelium proliferation.

\section{The effect of the mineral medium on the growth of Omphalotus af. illudent}

The investigation of rich organic media: peptone in concentration of $3 \%$ and higher, corn husks and soybean hulls in amounts of up to $30 \%$, which also served as nitrogen source, yielded the following results. As the amount of peptone in the nutrient medium reached $5 \%$, the rate of increase in mycelium biomass got higher. Similarly, as the percent of corn husks and soybean hulls in the nutrient medium was raised to 30 , the increase in fungus biomass became greater. Peptone concentrations higher than $6 \%$ and amounts of corn husks and soybean hulls larger than $30 \%$ of the nutrient medium were not tested because such media, excessively rich in organics, tend to become overgrown with other fungi, whose growth rates are higher than that of Omphalotus af. illudent.
Omphalotus af. illudent mycelium was grown in experiments with the media supplemented with different sources of nitrogen, including inorganic salts and urea: $\mathrm{NaNO}_{3}, \mathrm{KNO}_{3}, \quad\left(\mathrm{NH}_{4}\right)_{2} \mathrm{SO}_{4}$, and $\left(\mathrm{NH}_{2}\right)_{2} \mathrm{CO}$. Results of measurements are shown in Fig. 4. Our results suggest that the biomass increase is greater at enhanced nitrogen concentrations, but tolerance of Omphalotus af. illudent culture to the medium depends on the form of the added nitrogen. The lowest tolerance was observed to the urea-containing medium. Even at a urea concentration of $2 \%$ the growth was inhibited, and at $3 \%$ the culture stopped growing. A possible reason for this may be that at cultivation temperatures higher than $30^{\circ} \mathrm{C}$ urea is rapidly decomposed to ammonia.

We studied effects of potassium (KCL and $\left.\mathrm{K}_{2} \mathrm{CO}_{3}\right)$, phosphorus $\left(\mathrm{P}_{2} \mathrm{O}_{5}\right)$, and magnesium $\left(\mathrm{MgSO}_{4}\right)$ in the growth media. The effect of potassium added as a salt to the nutrient medium is shown in Fig. 5a. The results suggested that potassium concentration in the medium which was higher than $0.5 \%$ value which inhibits mycelial growth. The effect of the further increase in potassium concentration depended on whether $\mathrm{KCl}$ or $\mathrm{K}_{2} \mathrm{CO}_{3}$ was added. While an increase in $\mathrm{KCl}$ concentration to $4 \%$ did 


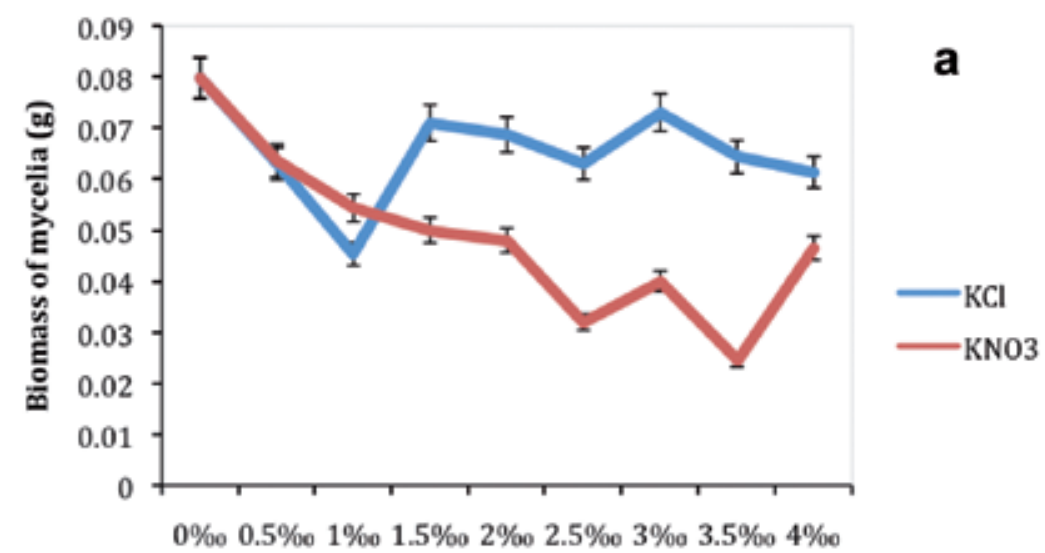

Potassium concentration $(\% 0)$

b
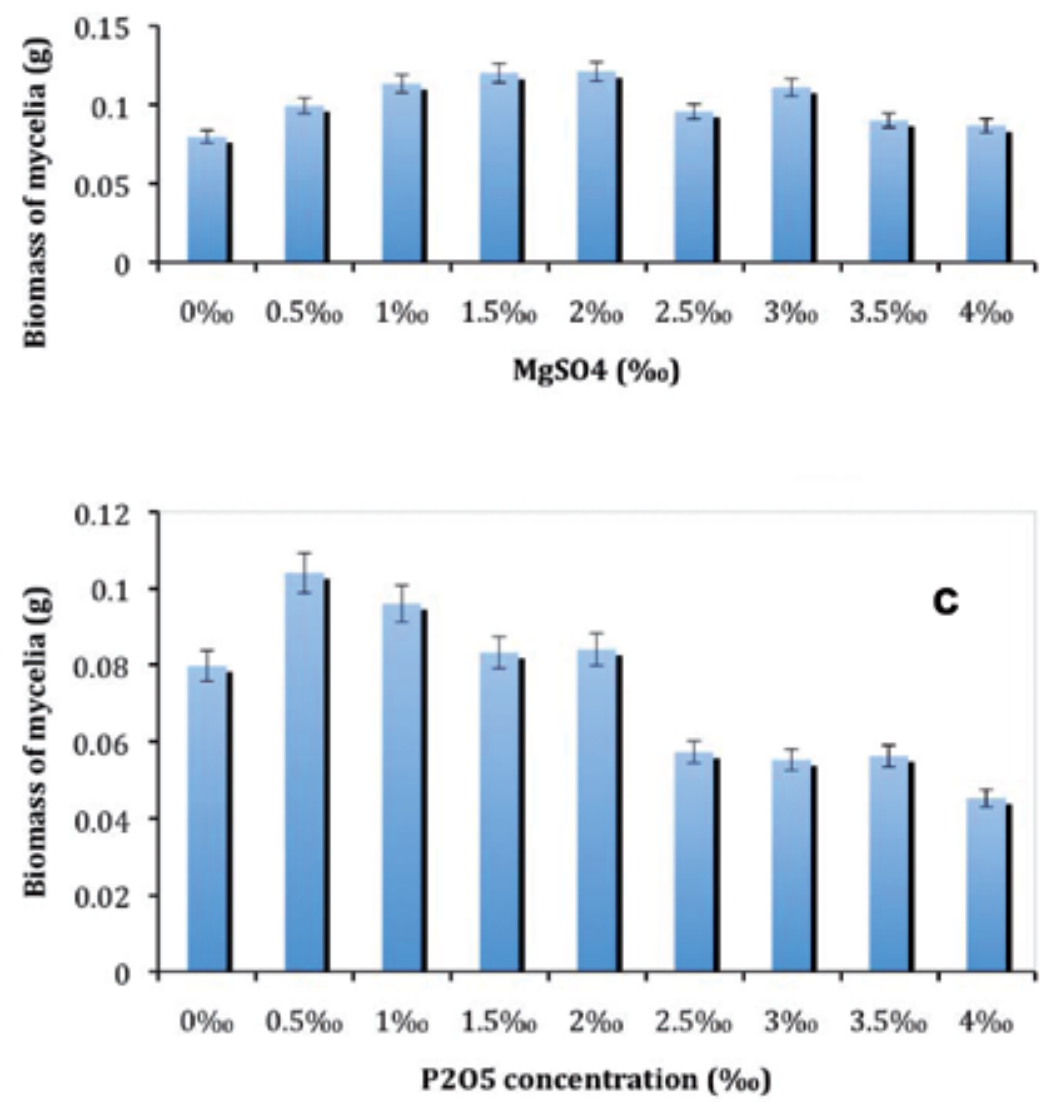

Fig. 5. Effect of addition of salts in the medium on mycelial growth rate a) potassium salts, b) magnesium sulfate, c) phosphorus oxide 
not cause a significantly greater decrease in the growth rate than that observed at the $0.5 \%$ potassium concentration, the increase in $\mathrm{K}_{2} \mathrm{CO}_{3}$ concentration consistently reduced the growth rate, probably due to rising alkalinity of the medium. Fig. 5b demonstrated the effect of $\mathrm{Mg}$ increase in the medium, caused by the addition of $\mathrm{MgSO}_{4}$. As shown, the best growth of mycelium was registered at $\mathrm{Mg}$ concentration of 1.5-2.0\%. At lower and higher Mg levels the growth was inhibited but was not slower than that in the $\mathrm{Mg}$-free medium. The optimal concentration of phosphorus added as $\mathrm{P}_{2} \mathrm{O}_{5}$ was 0.5-1.0 \%. Phosphorus concentrations higher than that inhibited mycelial growth (Fig. 5c). At $\mathrm{P}$ concentration of $4.0 \%$, the growth rate of mycelium was half of that at 0.5-1.0\%.

Results of experiments with mineral salts added to the medium suggest that Omphalotus af. illudent is oligophilous towards $\mathrm{K}, \mathrm{Mg}$, and $\mathrm{P}$ present in the medium.

\section{Enzymatic activity of Omphalotus}

af. Illudent mycelium on different substrates

Growth of Omphalotus af. illudent mycelium was studied in experiments with the media containing starch, carboxyl methyl cellulose, lignin, and casein. The nutrient medium was supplemented with the tested substrates in the amounts up to $1 \mathrm{~g} / \mathrm{L}$. Disintegration of the tested substrates was measured on Petri dishes with agar-containing medium as a linear increase in Omphalotus af. illudent mycelium, which was growing out of the center of the dish and had a regular round shape. Measurements were performed on the mycelium cultured for 3 days at the temperature $30{ }^{\circ} \mathrm{C}$.

In Fig. 6 photographs of Petri dishes with Omphalotus af. illudent mycelium growing on media supplemented with carboxyl methyl cellulose (a), casein (b), starch (c), and lignin (d) are presented. Fig. 7 shows the mean daily linear rate of the growth of Omphalotus af. illudent mycelium on these media. As Omphalotus af. illudent preferably grows on old and dying trees, this naturally resulted in that lignin was disintegrated at the highest rate and was followed by carboxyl methyl cellulose; disintegration of casein and starch were the slowest, although not zero.

IV. The effects of physical factors on growth and development of Omphalotus af. illudent

\section{IV.1 Temperature}

The increase in the Omphalotus af. illudent biomass was measured in the liquid culture grown on the maltose medium for 5 days at the temperature from $15{ }^{\circ} \mathrm{C}$ to $45{ }^{\circ} \mathrm{C}$, at $5{ }^{\circ} \mathrm{C}$ intervals. The average values for three replicated experiments are presented in Fig. 8a.

There was clear dependence of mycelium growth on the temperature of the liquid medium, with an obvious optimum at $30^{\circ} \mathrm{C}$, which corresponded to the most frequently registered temperature in the natural habitat of Omphalotus af. illudent in Vietnam. The temperature dependence was asymmetrical. At the temperature higher than the optimum in $5{ }^{\circ} \mathrm{C}$ the growth rate decreased for $25 \%$ and at $40{ }^{\circ} \mathrm{C}\left(10^{\circ} \mathrm{C}\right.$ higher than the optimum $)$ the growth nearly ceased. However, at the temperature below the optimum for $10{ }^{\circ} \mathrm{C}$, the growth rate was just $40 \%$ lower. Even at temperature of $15{ }^{\circ} \mathrm{C}$, which cannot be frequently registered at this latitude, mycelium kept growing - at the rate twice lower than the optimal.

Thus, there was a distinct temperature optimum for the growth of Omphalotus af. illudent, but it is not a stenothermal species. It can still grow at temperatures $15{ }^{\circ} \mathrm{C}$ lower than the optimal one, although its growth rate was half slowed down. 

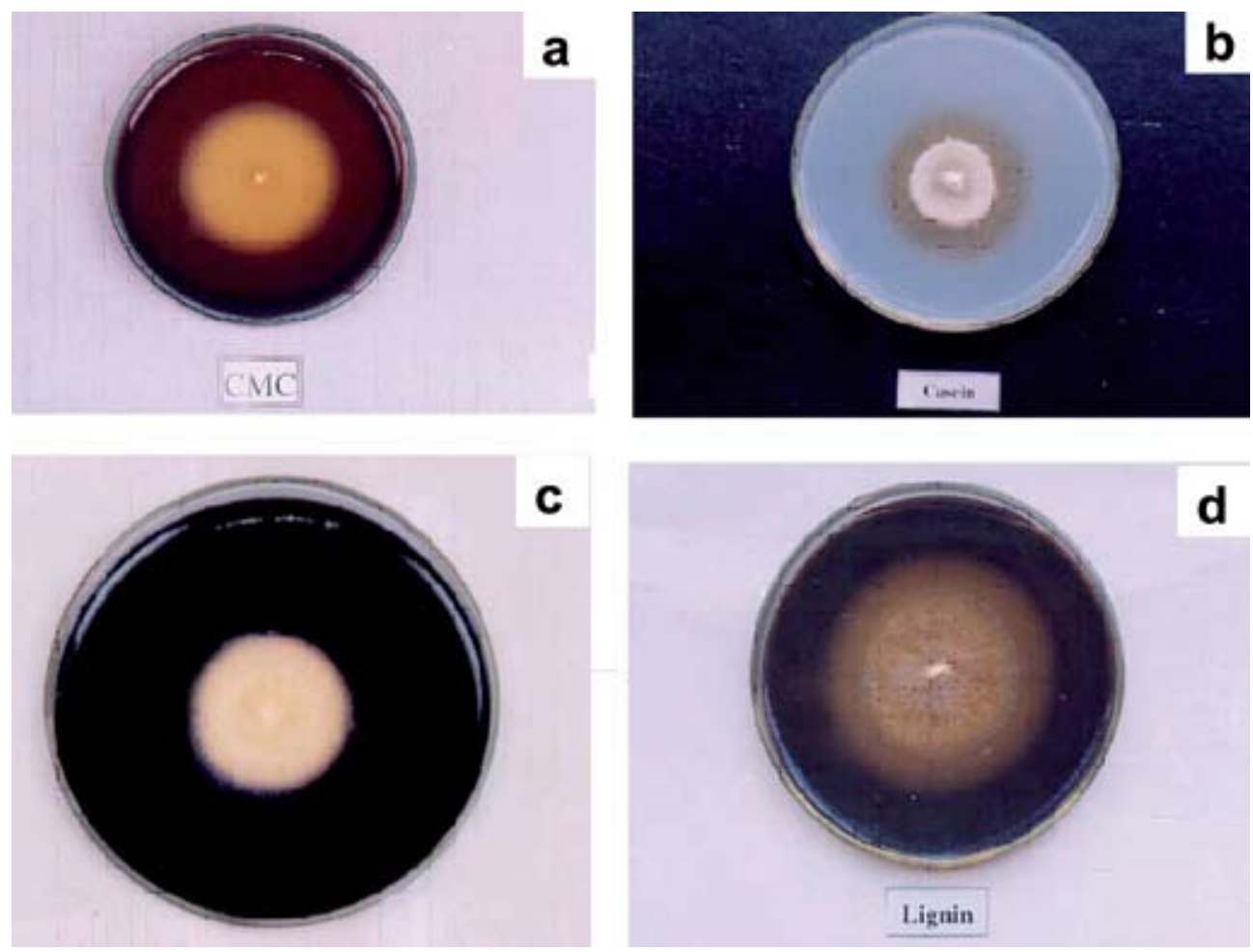

Fig. 6. Omphalotus af. illudent mycelium growing on: a - carboxyl methyl cellulose; b - casein; c - starch; $\mathrm{d}$ - lignin, concentration of each compound was $1 \mathrm{~g} / \mathrm{l}$

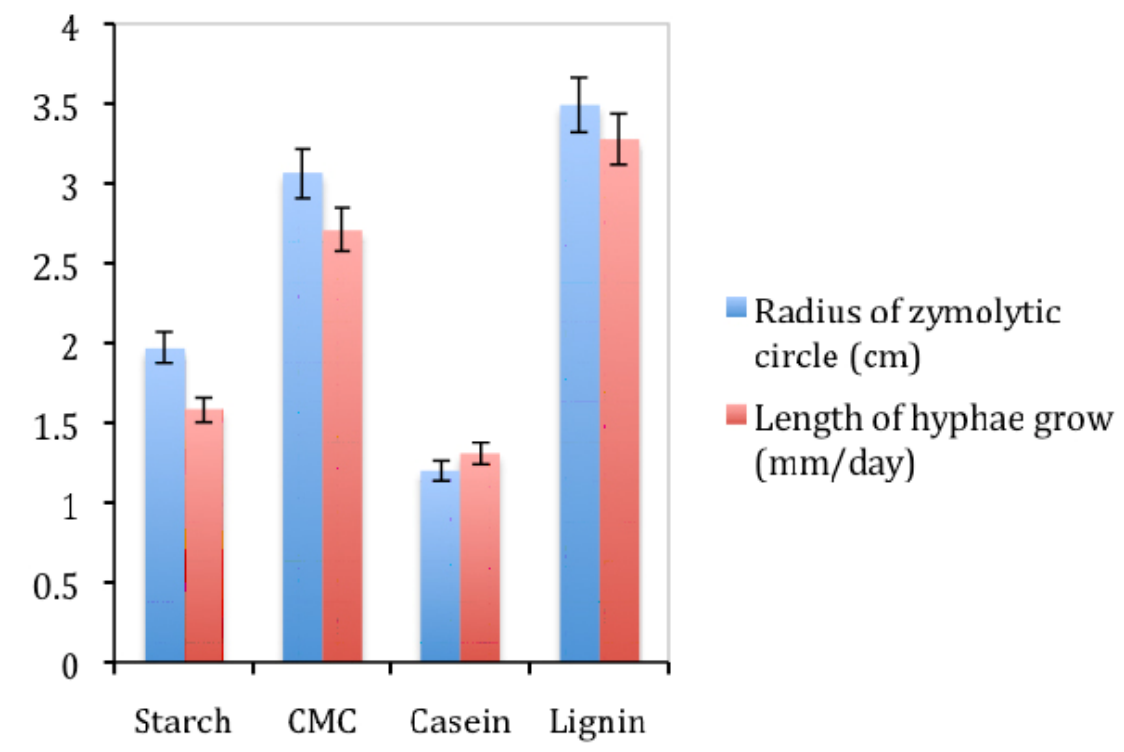

Fig. 7. Capacity of substrate disintegration of exocrine enzymes of Omphalotus af. illudent on carboxyl methyl cellulose, casein, starch and lignin 

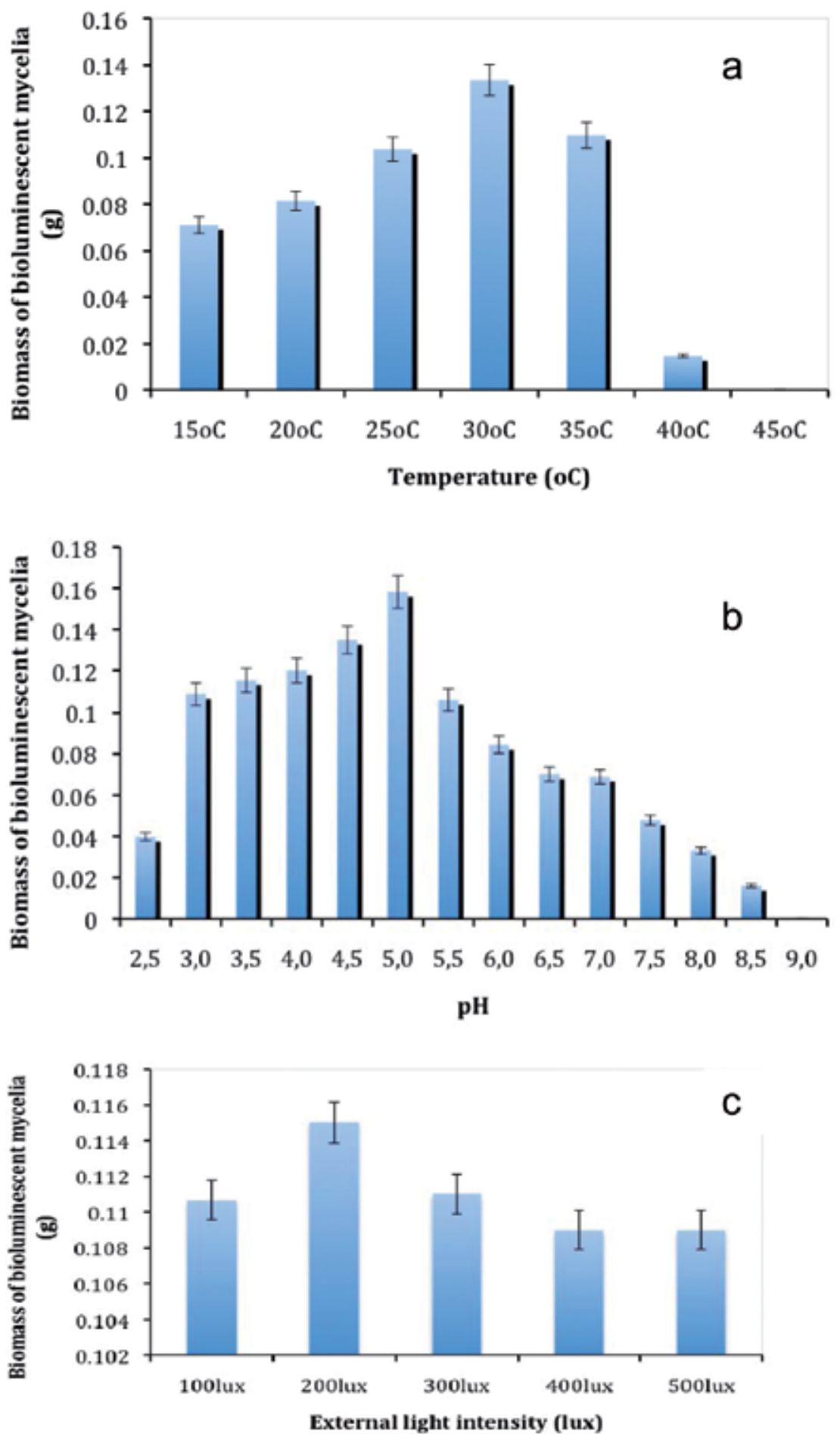

Fig. 8. Relationship of Omphalotus af. illudent biomass growth to a) temperature, b) pH, c) light 


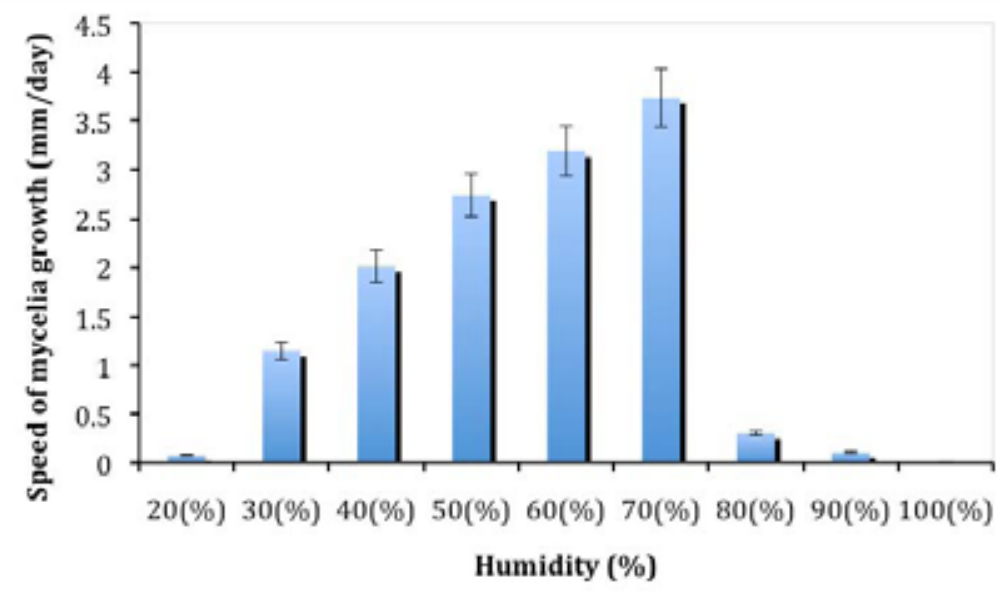

Fig. 9. The effect of humidity on the growth of Omphalotus af. illudent mycelium

\section{IV.2 $\mathrm{pH}$ of the medium}

We measured the biomass of mycelium grown at $\mathrm{pH}$ values varied from 3 to 7 , at constant temperature $30^{\circ} \mathrm{C}$. The experiment was replicated 3 times. The average values for three replicated experiments are presented in Fig. 8b.

Mycelium growth rate obviously depended on $\mathrm{pH}$ of the medium, with the maximum at $\mathrm{pH} 5$, a rapid decrease at more alkaline values, and a slower one at more acidic $\mathrm{pH}$ values. However, throughout the range from $\mathrm{pH} 3$ to 7 mycelium retained its ability to grow; at $\mathrm{pH} 3$ its growth rate was $75 \%$ of the highest one (at $\mathrm{pH} 5$ ) and at $\mathrm{pH} 7$ it dropped to $47 \%$. Thus, Omphalotus af. illudent grows best in weakly acidic conditions, which should be maintained in the cultures of this fungus.

\section{IV.3 Light}

In this experimental series Omphalotus af. illudent was grown on maltose under different lighting conditions. Luminance from 100 to 300 lux did not produce any consistent effect on mycelium growth rate, which was the same as the growth rate in the dark. A noticeable growth inhibiting effect was only observed at higher light conditions - 400-500 lux. Formation of fruiting bodies seemed to be triggered by the illuminance 500-800 lux (Fig. 8c).
As Omphalotus af. illudent emits light of its own, its relationship to the ambient light is of considerable interest and is worthy of more profound research.

\section{4 Humidity}

The level of humidity must be the most significant limiting factor that determines the growth and development of the Omphalotus af. illudent fungus in tropical forests of Vietnam. The effect of humidity on the growth of Omphalotus af. illudent mycelium was studied by measuring mycelium linear growth, $\mathrm{mm} / \mathrm{d}$, on rubber tree sawdust media.

Results presented in Fig. 9 show that humidity levels between $30 \%$ and $70 \%$ served as a limiting factor for the growth of Omphalotus af. illudent mycelium. As the level of humidity rises from $30 \%$ to $70 \%$, the growth rate increase reached $300 \%$. However, higher levels of humidity, $80 \%-90 \%$, terminated the growth of mycelium and triggered the formation of fruiting bodies.

\section{Bioluminescence of Omphalotus af. illudent}

A specific feature of Omphalotus af. illudent metabolism is bright visible wavelength luminescence. It is observable at a distance of 


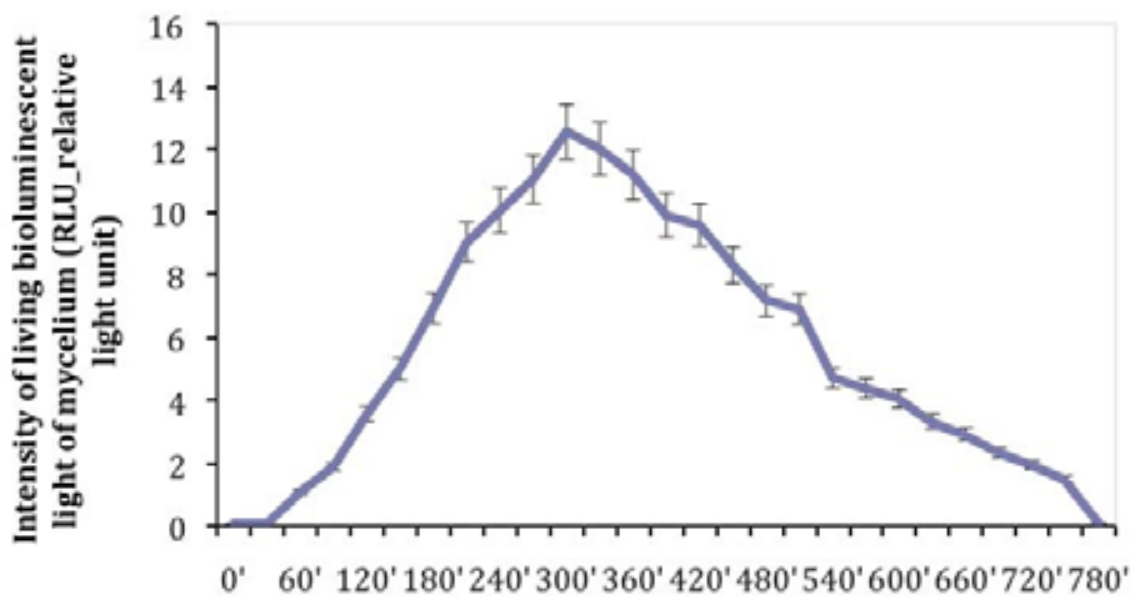

Time (minute)

Fig. 10. Luminescence intensity of Omphalotus af. illudent mycelium
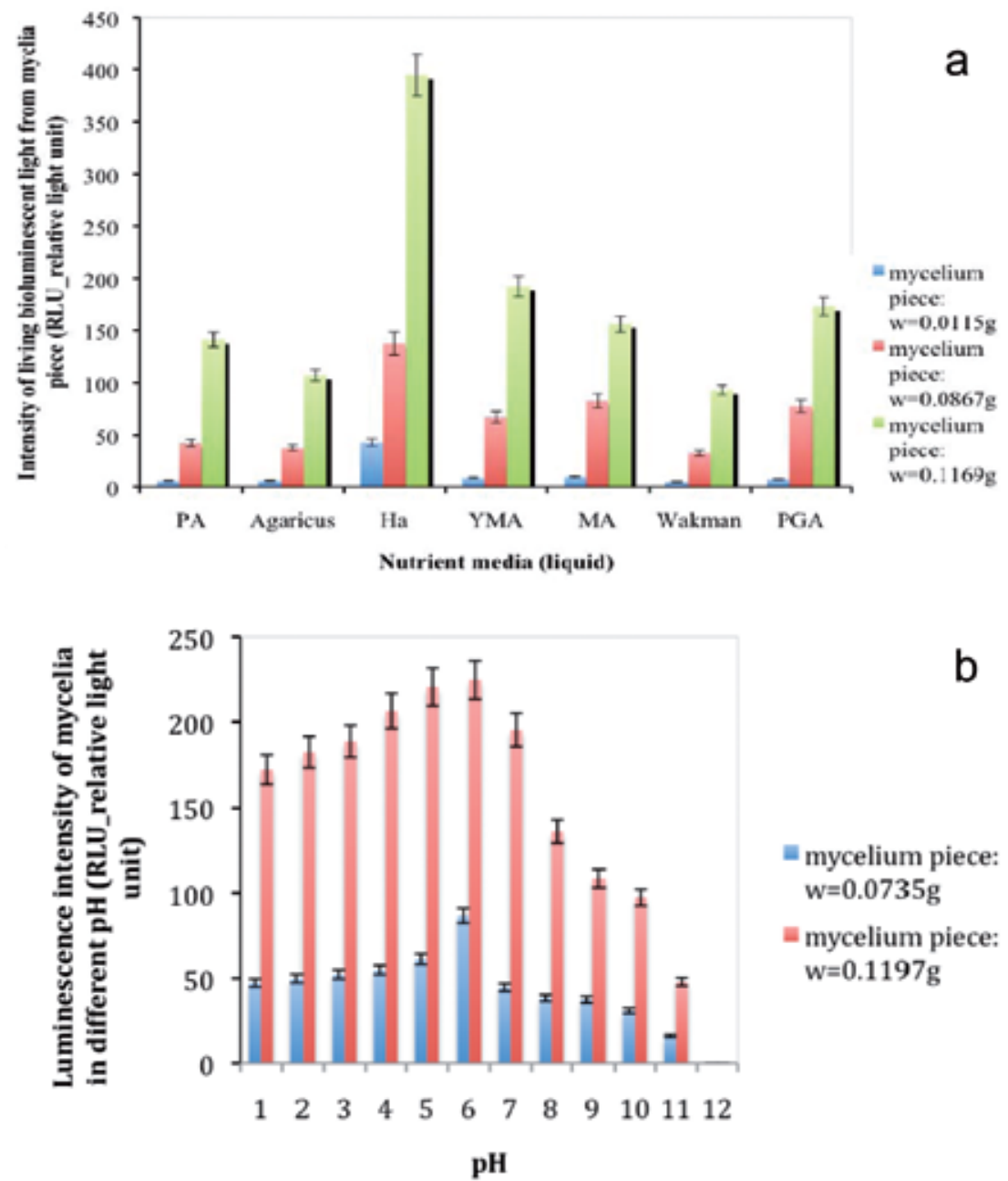

b

= mycelium piece: $\mathrm{w}=\mathbf{0 . 0 7 3 5 \mathrm { g }}$

= mycelium piece: $\mathrm{w}=0.1197 \mathrm{~g}$

Fig. 11. Relationship of luminescence intensity of Omphalotus af. illudent to: a) type of nutrient media, b) pH of the medium 


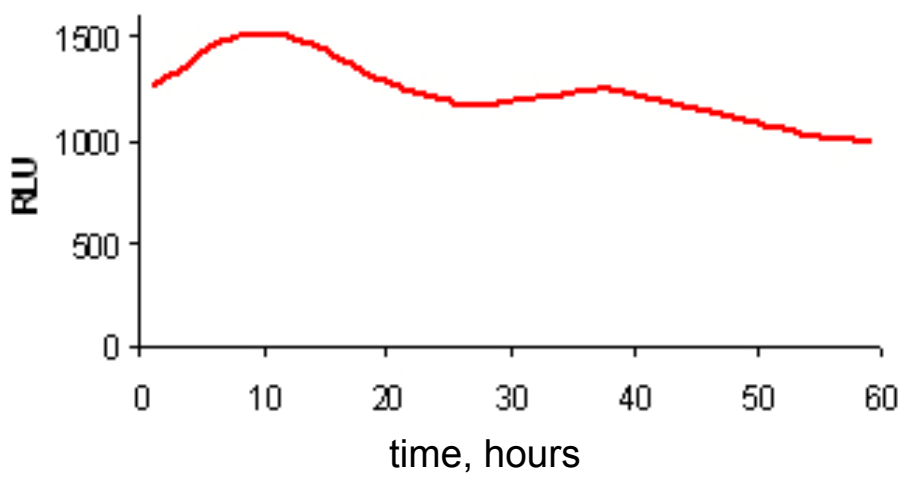

Fig. 12. Dynamics of intensity of luminescence of young fruiting body of Omphalotus af. illudent (budding 3 days, weight $0.914 \mathrm{~g}$, moisture $87.76 \%$, $\mathrm{pH} 8.5$ )

several meters and can easily be registered by luminometers. In contrast to many other luminous fungi, this species is luminescent in every phase of its life cycle: from spores to fruiting bodies.

Omphalotus af. illudent luminescence intensity was measured using a laboratory prototype luminometer designed and constructed at Ho Chi Minh City University, Vietnam. Results of the discrete measurements are presented in relative luminescence units (RLU), in a graph of luminescence intensity for $13 \mathrm{~h}$ (Fig.10).

Luminescence intensity was studied in relation to the composition of the growth medium (Fig. 11a). Luminescence intensity on Hamada medium was considerably, 4-5 times, higher than that on other studied media. It is notable that the growth rate on this medium was the lowest. Thus, the processes of growth and luminescence in the fungus are reciprocal.

The relationship of the mycelium luminescence intensity to $\mathrm{pH}$ of the medium (Fig. 11b) was similar to the corresponding relationship of the growth rate: the maximum was observed at $\mathrm{pH}$ 7-8, but in much more acidic media, even at $\mathrm{pH} 1-3$, the ability of the mycelium to emit light was retained as well as its ability to grow. The $\mathrm{pH}$ dependence of the luminescence of the fruiting bodies is the same: its peak is registered at $\mathrm{pH}$ 6-7 and the ability to emit light is retained within a wide range of $\mathrm{pH}$ values - from 1 to 11, i.e., from strongly acidic to strongly alkaline conditions, with the intensity symmetrically decreasing from the optimum.

Long-duration, lasting several tens of hours, measurements of luminescence, which were taken every $10 \mathrm{~min}$, revealed wavelike variations in the luminescence intensity of three-day-old fruiting bodies (Fig. 12). Oscillations of fungal luminescence have been previously reported by other authors (Berliner, 1961; Calleja and Reynolds, 1970; Kamzolkina,1982).

The revealed relationship between the luminescence rhythm and the age of fruiting bodies should be investigated further.

\section{The life cycle of the Omphalotus}

\section{af. illudent fungus}

In natural optimal conditions, growing on the wood of the rubber tree (Hevea sp.), the development of Omphalotus af. iludent from spore to spore lasts up to 2 months, with the following duration of different phases:

Spore germination and onset of growth -5 days.

Spread of mycelium in the wood substrate 20 days.

Formation of hyphae inside the wood -20 days. 


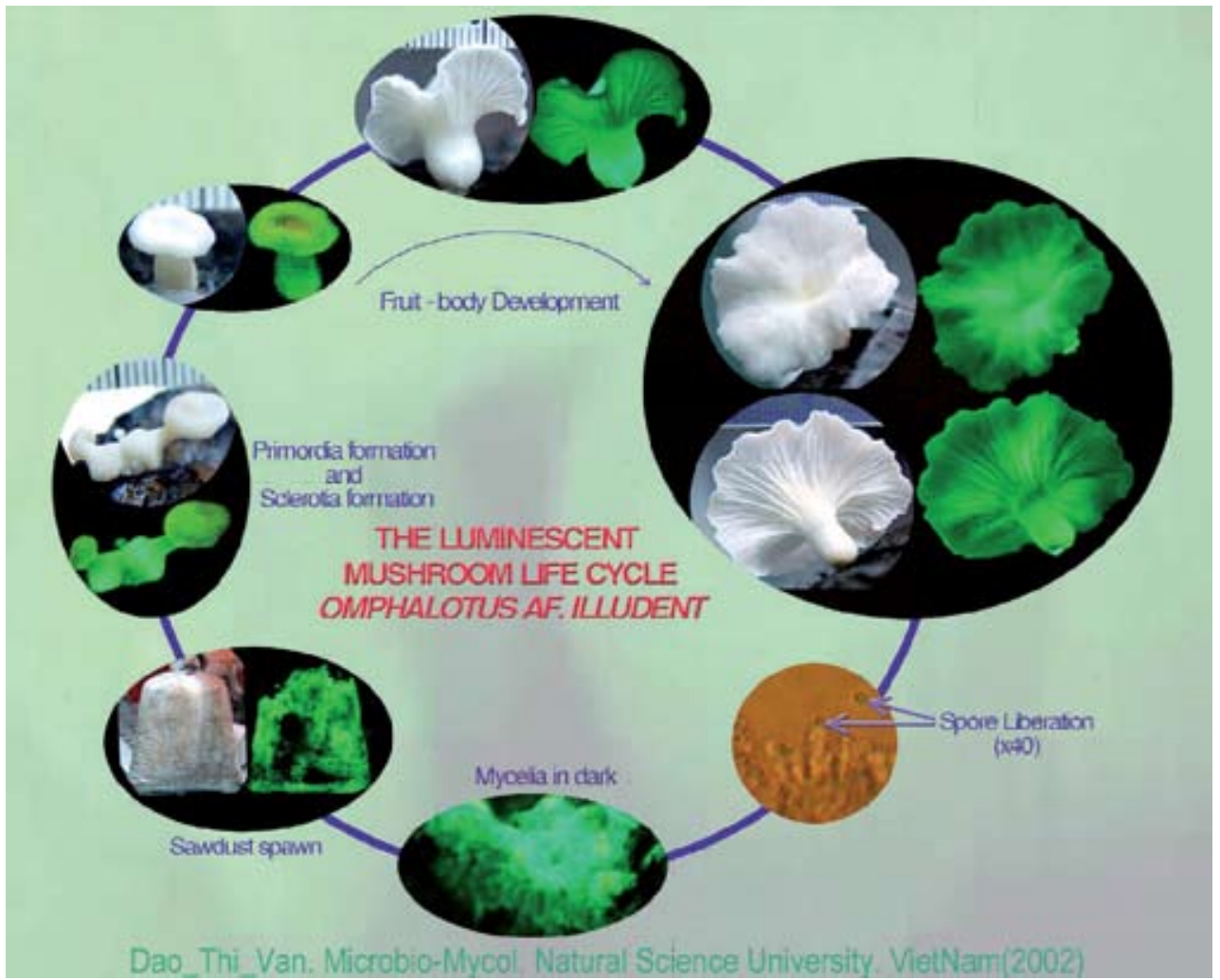

Fig. 13. The life cycle of Omphalotus af. illudent in nature

Fruiting body formation and maturation -15 days.

The switching from mycelium growth to the formation of fruiting bodies was found to be triggered by a sharp change in the temperature of the air $\left( \pm 5^{\circ} \mathrm{C}\right)$ and/or a dramatic, usually postrain, increase in the humidity - to $90 \%$. The life cycle of Omphalotus af. illudent in nature is shown in a series of photographs in Fig. 13.

The study of the physiological and cultural properties of Omphalotus af. illudent provided a basis for successful large-scale cultivation of this fungus and, hence, extraction of products of potential pharmaceutical significance from the biomass in quantities sufficient for studies of the mechanism underlying fungal bioluminescence and for use the fungus in bioluminescent assay.

The author is grateful to Mr. Le Duy Thang, Natural Science University, Mr. Nguyen Vu, Natural Science University for collecting fungus samples in field and for technical assistance.

\section{References}

Berliner M. D. (1961) Studies in fungal luminescence. Mycologia 53: 84.

Bitler B., McElroy W.D. (1957) Preparation and properties of crystalline firefly luciferin, Arch. Biochem. Biophys., 72: 358.

Calleja G. B., Reynolds G. T. (1970) The oscillatory nature of fungal bioluminescence. Trans. Br. Mycol. Soc. 55: 149.

Desjardin D. E., Capelari M., Stevani C. V. (2005) A new bioluminescent agaric from S ao Paulo, Brazil. Fung. Div. 18: 9. 
Desjardin D. E., Capelari M., Stevani C. V. (2007) Bioluminescent Mycena species from S ao Paulo, Brazil. Mycologia 99: 317.

Desjardin D. E., Braga-Neto R. (2007) Mycena lacrimans, a rare species from Amazonia, is bioluminescent. Edinburgh J. Bot. 64: 275.

Desjardin D.E., Oliveira A.G., Stevani C.V. (2008) Fungi bioluminescence revisited. Photochem. Protobiol. Sci. 7 : 170-182.

Dubois R. (1885) Note sur physiologie des Pyrophores. C. R. Seances Soc. Biol. Paris, 2 : 559.

Kamzolkina O. V. (1982) Oscillation of bioluminescence in submerged culture of Armillaria mellea (Fr.) Kumm., Mikologiya i Fitopatologiya 16: 323.

Kirchmair M., Pëder R., Huber C.G., Miller O.K. (2002) Chemotaxonomical and morphological observations in the genus Omphalotus Fayod (Omphalotaceae). Persoonia 17: 583.

Shimomura O. (1992) The role of superoxide dismutase in regulating the light emission of luminescent fungi. J. Exp. Bot. 43: 1519.

Shimomura O. (2006). Bioluminescence: Chemical Principles and Methods. London, World Scientific Publishing Co. 500 p. 